\title{
Polarized Galactic Emission Mapping: helping to unfold the veil of the Cosmic Microwave Background
}

\section{Domingos Barbosa ${ }^{1}$}

Centro de Física da Universidade do Porto

Porto, Portugal

E-mail: dsbarbos@fc.up.pt

and

CENTRA, Instituto Superior Técnico, Lisboa, Portugal

\section{Rui Fonseca}

Instituto de Telecomunicações, Campus Universitário de Santiago,

Aveiro, Portugal

\section{Dinis M. dos Santos}

Dep. Electrónica e Telecomunicações, Campus Universitário de Santiago,

Aveiro, Portugal

\section{Luis Cupido}

Centro de Fusão Nuclear, Instituto Superior Técnico,

Lisboa, Portugal

\section{Ana Mourão}

CENTRA, Instituto Superior Técnico,

Lisboa, Portugal

\section{George F. Smoot}

\section{${ }^{1}$ Speaker}


Astrophysics Group, Lawrence Berkeley National Laboratory,

Berkeley, USA

\section{Camilo Tello, Ivan Soares, Thyrso Villela}

Instituto Nacional de Astrofísica Espacial, Div. Astrofisica,

São José dos Campos SP, Brazil

\section{Marco Bersanelli}

Physics Department, Uni. Milano, Italy

In the context of the new era of Cosmic Microwave Background Polarization (CMBP) experiments, it is important to have reliable templates of the polarized foregrounds contaminating the CMBP. This is specially true for the for the Planck Surveyor Mission (ESA, 2007) and for the near-future B-mode polarization probes. The Galactic Emission Mapping collaboration aims to measure the polarization state and Stokes components at low frequencies (5$10 \mathrm{Ghz}$ ) of the galactic synchrotron radiation using an antenna covering the North Hemisphere and another the South Hemisphere. Together, the joint coverage should produce a reliable template of the synchrotron polarization in $80 \%$ of the sky.

European Physical Society

HEP2005 International Europhysics Conference on High Energy Physics

EPS (July $21^{\text {st }}$-27 $7^{\text {th }}$ 2005) in Lisboa, Portugal

\section{The Galactic Emission Mapping collaboration}

Experiments like Planck Surveyor will require well estimated templates of polarized synchrotron (affecting the Planck Low Frequency Instrument $30-90 \mathrm{GHz}$ ) and polarized dust emission (affecting the Planck High Frequency Instrument 100-800 $\mathrm{GHz}$ ). Existing polarization templates (for low frequencies - synchrotron) rely on a semi-empirical approach of extrapolating polarization substructure information from small sky area surveys with theoretical assumptions of depolarization of the synchrotron observed templates [4]. Furthermore, correlations of the WMAP first-year data with available foreground templates show another physical component appearing to be important - spinning dust emission which was not accounted for in the data processing analysis [5]. Thus, with foreground estimation as one of the main tasks currently being pursued by CMB teams, the Galactic Emission $\operatorname{Mapper}^{1}$ (GEM) -was upgraded towards polarized foreground cartography at $5 \mathrm{GHz}$ (C. Tello, in preparation) with the development of a new high-sensitivity pseudo-correlator

${ }^{1}$ USA site: http://aether.lbl.gov/www/projects/gem

Brazil site : http://www.cea.inpe.br/ cosmo/GEM/index gem.htm 
receiver. It will map the polarized synchrotron radiation (polarized at a maximum of $\sim 60 \%$ level) where this emission is dominant down to a sensitivity of $0.5 \mathrm{mK}$.
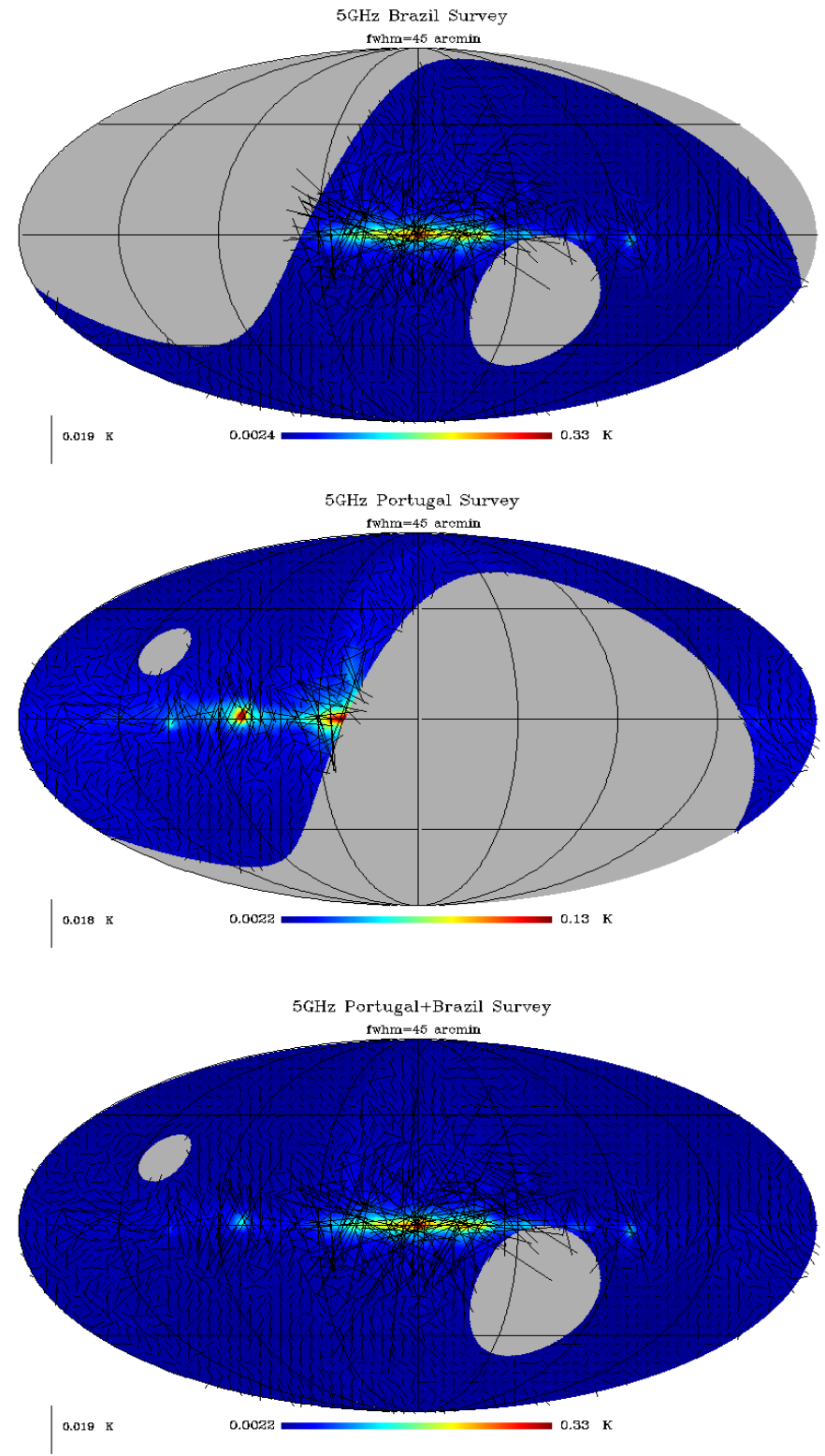

Fig.1 - Simulated synchrotron maps at $5 \mathrm{Ghz}$. The top figure represents the South Hemisphere coverage (Brasil antenna), the middle figure represents the North Hemisphere coverage (Portugal antenna) and the bottom figure is the joint coverage. All maps have a resolution of 45 arcmin.

At the same time, continuous work on the receiver will prepare the upgrades towards $10 \mathrm{GHz}$ observations, where some signal from spinning dust is expected to appear mixed to synchrotron emission. At a later stage, by 2007, GEM-Brazil and GEM-Portugal maps will be merged to produce the biggest 
sky templates of the polarized synchrotron components ( $~ 80 \% \mathrm{sky})$. These templates will shed light on the synchrotron polarization fraction and substructure at scales down to 0.5 o, covering a range of scales important for B-mode searching and allowing a better polarized foreground subtraction from large sky data sets such as the Planck Surveyor maps.

\section{Acknowledgments}

This work was supported by FCT- Portugal through projects POCTI/FNU/42263/2001 and POCI/CTE-AST/57209/2004 and by FAPESP Brasil.

\section{References}

[1] C. Tello et al., Spillover and diffraction sidelobe contamination in a doubleshielded experiment for mapping Galactic synchrotron emission, Astronomy \& Astrophys., 145, 495 (2000)

[2] Fonseca et al., Site Evaluation and RFI spectrum measurements in Portugal at the frequency range 0.408-10 GHz for a GEM polarized galactic radio emission experiment, New Astronomy, astro-ph/0411477 (2004)

[3] S. Torres et al., The GEM project: an international collaboration to survey galactic radiation emission, Astroph. Space Sci., 240, 225 (1996)

[4] G. Girardino et al., Towards a model of full-sky Galactic synchrotron intensity and linear polarisation: A re-analysis of the Parkes data, Astronomy \& Astroph., 387, 82, (2002)

[5] D. Finkbeiner, Microwave Interstellar Medium Emission Observed by the Wilkinson Microwave Anisotropy Probe, Astrophys.J., 614, 186-193 (2004). 